\title{
Curvature-based Curb Detection Method in Urban Environments using Stereo and Laser
}

\author{
C. Fernández, D. F. Llorca, C. Stiller, M. A. Sotelo
}

\begin{abstract}
This paper addresses the problem of curb detection for ADAS or autonomous navigation in urban scenarios. The algorithm is based on clouds of $3 \mathrm{D}$ points. It is evaluated using 3D information from a pair of stereo cameras and a LIDAR. Curbs are detected based on road surface curvature. The curvature estimation requires a dense point cloud, therefore the density of the LIDAR cloud has been augmented using Iterative Closest Point (ICP) based on the previous scans. The proposed algorithm can deal with curbs of different curvature and heights, from as low as $3 \mathrm{~cm}$, in a range up to $20 \mathrm{~m}$ (whenever that curbs are connected in the curvature image). The curb parameters are modeled using straight lines and compared to the ground-truth using the lateral error as the key parameter indicator. The ground-truth sequences were manually labeled on urban images from the KITTI dataset and made publicly available for the scientific community.
\end{abstract}

\section{INTRODUCTION AND RELATED WORK}

Road detection has been a topic of the utmost relevance in the fields of Advanced Driver Assistance Systems (ADAS) and autonomous driving. In the last years, the quality and performance of ADAS has improved significantly, providing a crucial contribution for making autonomous driving a reality. Nowadays, some production vehicles are equipped with systems that take the control of the vehicle in order to reduce the damage to the occupants in case of accident. All these systems require accurate scene understanding capability. For such purpose, vision sensors have been extensively used by the automotive industry in diverse systems. Lane departure warning (LDW) and lane keeping systems are clear examples of that. These types of systems are consolidated in today's cars. However their use is limited to highways and roads with clearly visible lane markers. Robust operation of LDW systems on unmarked roads and urban environments is still a challenge. In most non-urban roads, lanes are limited by road markings. However, metropolitan areas are more complex and the free space can be restricted by road markings, parked cars, traffic signs, lampposts and curbs of quite different heights. The accuracy and reliability of vision based systems are strongly affected by the large variety of street configurations, different materials and textures, illumination changes, etc.

In order to deal with this adversities, research resources must be oriented to developing algorithms for the reliable detection of curbs and road edges, see Figure 1. This paper

C. Fernández, D. F. Llorca and M. A. Sotelo are with the Computer Engineering Department, Polytechnic School, University of Alcalá, Madrid, Spain. email: carlos.fernandez, llorca, sotelo@aut.uah.es. Christoph Stiller is with the Institute of Measurement and Control Systems at Karlsruhe Institute of Technology, Karlsruhe, Germany. email: stillerakit.edu focuses on curb detection on urban scenarios. We propose a versatile curvature-based method which can operate using dense $3 \mathrm{D}$ point clouds regardless the specific sensor. Thus, our approach has been validated using 3D data captured from stereo vision and LIDAR. Free space is crucial for understanding the road scene, providing relevant information to the path planning module in an autonomous vehicle.

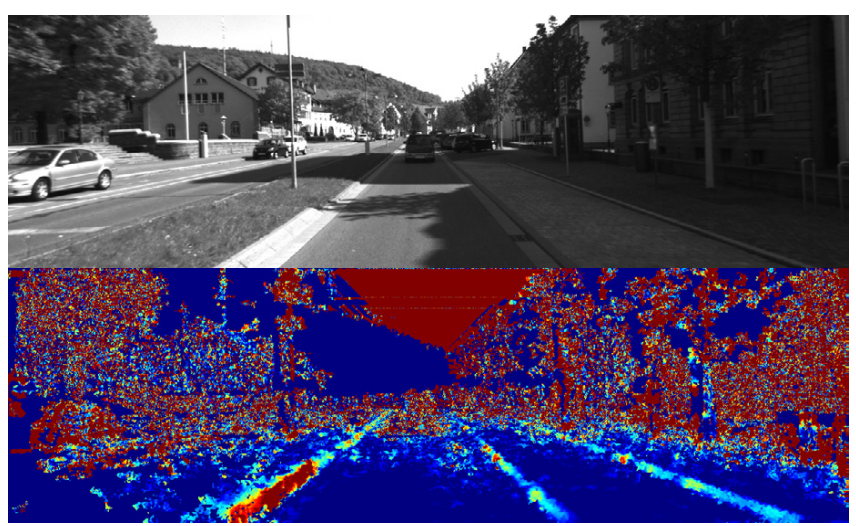

Fig. 1. Top: example of a complex urban scenario. Bottom: the curvature representation. On the left side, the scene has a high curb in the closer distance and a lower one further. On the right side, there are two small curbs, the first one is the boundary for the parking place and the second one is the limit of the sidewalk.

In situations where no lane markings or curbs are present in the scene, texture and color information are inherent features to detect the road edges. The HSI color space is used in [1] and [2] together with road shape restrictions. An important issue for vision-based systems is the presence of shadows in the scene. In [3] an illuminant invariant image is obtained to remove the influence of the light changes. This illuminant invariant image is applied to road detection in [4]. In [5], firstly, patches are extracted from monocular images and later on analyzed to find local visual properties for a metric confidence map. Secondly, features called SPatial RAY (SPRAY) are computed on the confidence map. Afterwards, the ego-lane is extracted applying a semantic segmentation based on a local classification of the SPRAY features. In other cases LIDAR is used for enhancing visionbased systems. In [6], the authors propose a free space and speed humps detection system based on LIDAR and a monocular camera. Generally, monocular based systems are less robust than stereo-vision for road structure reconstruction. Consequently, in [7] a semantic graph associated with a stereo-based homography is proposed, where the road edges are located using the Viterbi algorithm. As a 
continuation of the previous work [8], the authors propose to solve the homography as a maximum a posteriori (MAP) problem in a Markov Random Field (MRF) that computes the binary labels road/non-road, learning the optimal parameters for the probabilistic algorithm. The matching algorithm for computing the disparity image is an important stage of a stereo vision system. As a matter of fact, disparity mismatching leads to unexpected results. In order to compensate for that, in [9] the authors propose a model of arbitrarily oriented slanted planes aiming at improving the road surface estimation. In [10], Loopy Belief Propagation is used to assign points extracted from the $3 \mathrm{D}$ point cloud to curb adjacent surfaces. As a consequence, the reconstruction of the curbs can be done even in low height curbs up to 20 meters. An improvement of the previous work is presented in [11], where the authors include a temporal filter to improve the accuracy and robustness. More recently, a system has been deployed in [12] based on the sole use of vision, radar, and accurate digital maps for autonomous driving on all types of scenarios, including rural roads, small villages and major cities. For such purpose, visual clues were off-line obtained and learned in order to develop a robust self-localization system given the ego-pose, the road structure was extracted from a highly accurate digital map. The system successfully traversed more than $100 \mathrm{~km}$ in driverless mode along German roads and cities.

In this paper we propose an algorithm for online road curb detection based on curvature surface estimation in 3D point clouds. The clouds can be captured from different types of sensors, for example LIDAR or stereo cameras. The point cloud from LIDAR is sparse because of the vertical resolution. In order to increase the cloud density, an iterative algorithm is applied to align the latest scans. The proposed method is included in a more complex system for urban scene understanding with particular emphasis on unmarked roads. The rest of the paper is structured as follows: section II presents a general description of the system, including the methods for curvature estimation, curb detection and point cloud upsampling. Results and discussion are presented in section III. Finally, we analyze our conclusions and future work in section IV.

\section{SYSTEM DESCRIPTION}

\section{A. General Description}

In this paper, a curb detection method based on surface curvature is compared using two different inputs. The first one is the $3 \mathrm{D}$ reconstruction point cloud using the Semi Global Matching (SGM) algorithm based on stereo vision [13]. The second one is the $3 \mathrm{D}$ reconstruction point cloud obtained from Velodyne. Since we focus on urban environments, the performance of the system is evaluated using the public dataset: KITTI Vision Benchmark Suite. [14]. The dataset provides images and information of urban scenarios from different types of sensors, such as monochrome and color cameras, multilayer LIDAR, GPS and IMU. The technical characteristics of the sensors used in this paper are: 2 Grayscale cameras 1.4 Mpx (Point Grey Flea 2) and a laserscanner (Velodyne HDL 64E). The height of the cameras is 1.65 meters and the height of the LIDAR is 1.73 meters. Furthermore, the LIDAR is installed $27 \mathrm{~cm}$ behind the cameras. Consequently, the relationship between camera and LIDAR poses must be calibrated. The accuracy of the stereo-based 3D point cloud is reasonable high even for long distances due to the long stereo baseline $(0.54 \mathrm{~m})$ and the high resolution of the cameras. On the one hand, road scene reconstruction from stereo is more dense than LIDAR reconstruction but it is also affected by mismatching errors. On the other hand, LIDAR provides low noise measurements even at long range while at large distances the $3 \mathrm{D}$ reconstruction is sparse and dependant on the vertical resolution.

\section{B. Curvature Estimation}

The proposed curb detection method is based on surface curvature estimation presented in [15]. This feature has been also used in [16] and [17] for free space detection. The curvature describes the variation along the surface normal and it varies between 0 and 1 , where low values correspond to flat surfaces. The curvature feature is more robust and stable than tangent plane normal vectors. For each point $p$, the nearest neighbors (NN) $p_{i}$ in a surrounding area defined by a radius $R$ are selected. These points are used to create a weighted covariance matrix, where $k$ denotes the number of NN.

$$
\begin{gathered}
\bar{p}=\frac{1}{k} \sum_{i=1}^{k} p_{i} ; \mu=\frac{1}{k} \sum_{i=1}^{k}\left|p-p_{i}\right| \\
w_{i}=\left\{\begin{array}{lc}
\exp \left(-\frac{\left(p-p_{i}\right)^{2}}{\mu^{2}}\right) & \text { if }\left|p-p_{i}\right| \geq \mu \\
1 & \text { otherwise }
\end{array}\right. \\
C=\sum_{i=1}^{k} w_{i} \cdot\left(p_{i}-\bar{p}\right) \cdot\left(p_{i}-\bar{p}\right)^{T}
\end{gathered}
$$

The eigenvector $V$ and eigenvalues $\lambda$ of $C$ are computed as $C \cdot V=\lambda \cdot V$. A curvature measure $\gamma_{z}^{p}$ is defined by equation 4, where $\lambda_{x} \leq \lambda_{y} \leq \lambda_{z}$ are the eigenvalues of the covariance matrix $C$.

$$
\gamma_{z}^{p}=\frac{\lambda_{z}}{\lambda_{x}+\lambda_{y}+\lambda_{z}}
$$

\section{Curb Detection}

In our reference system, the $Z$ axis is orthogonal to the road, therefore the curvature $\gamma_{z}$ provides a discriminative descriptor of the road shape. Curb height and curvature $\gamma_{z}$ are highly correlated. Consequently, after thorough observation of urban scenes in the KITTI dataset, a set of thresholds $\alpha_{i}=\{1 \ldots N\}$ is used to label the type of curb, see Table I:

Curb curvature is different in each scene. For example, if the curb is a regular one, most of the points exhibit curvature values $\gamma_{z} \in\left[\alpha_{2}, \alpha_{3}\right)$, but there are also some curb points yielding significantly different values, see Figure 2(b). These measurement outliers are removed by means of a filtering process. A binary mask is applied for each curvature 


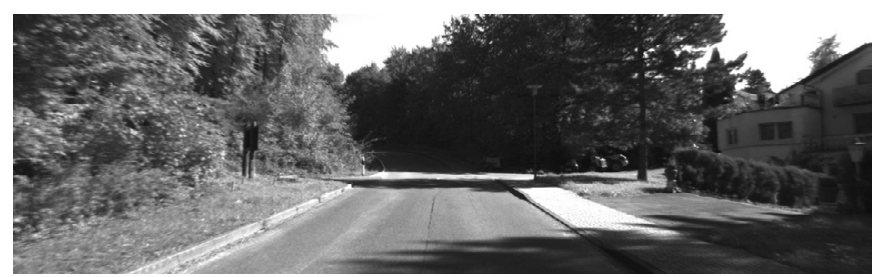

(a) Input image

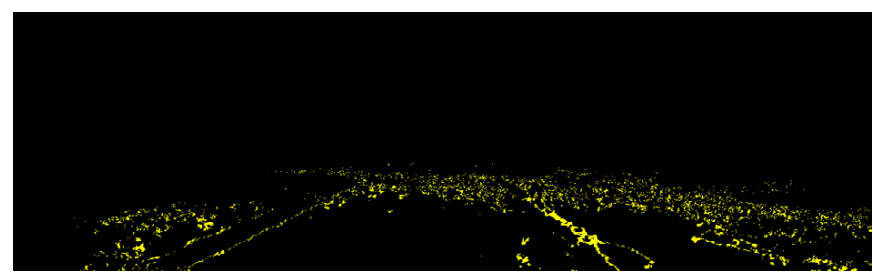

(c) Unfiltered mask for $\gamma_{z} \in\left[\alpha_{0}, \alpha_{1}\right)$

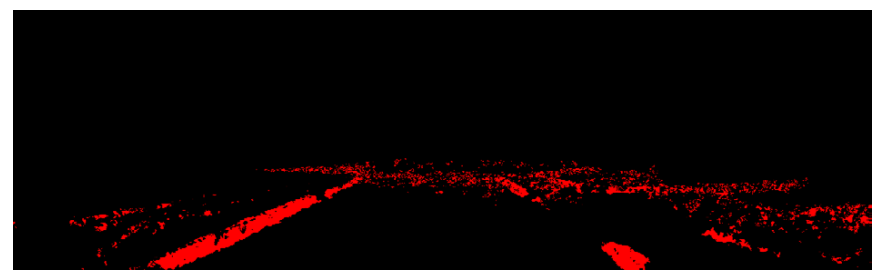

(e) Unfiltered mask for $\gamma_{z} \in\left[\alpha_{2}, \alpha_{3}\right)$

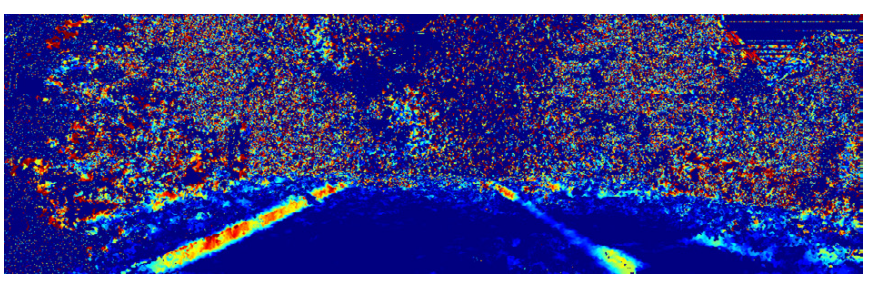

(b) Unfiltered curvatures

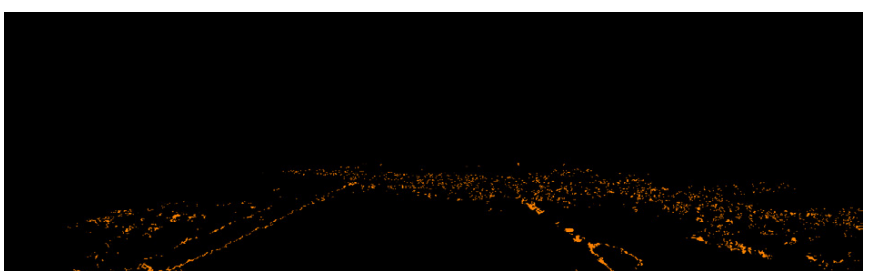

(d) Unfiltered mask for $\gamma_{z} \in\left[\alpha_{1}, \alpha_{2}\right)$

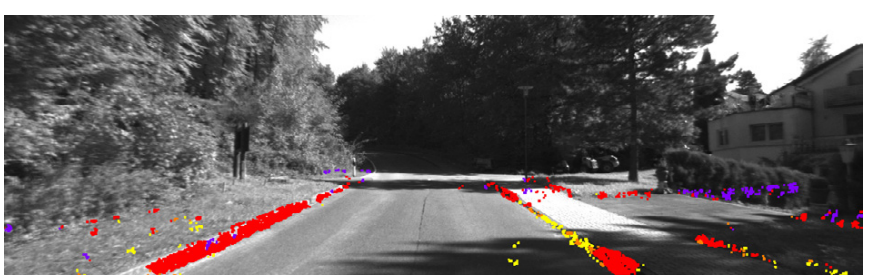

(f) Filtered curvatures

Fig. 2. Filtering process of the noisy input curvatures and the final result.

TABLE I

Curb Curvature Values

\begin{tabular}{|l|c|c|}
\hline DESCRIPTION & CURVATURE & COLOR \\
\hline Flat surface & $0 \leq \gamma_{z}<\alpha_{0}$ & not painted \\
\hline Very Small Curbs $(\sim 3 \mathrm{~cm})$ & $\alpha_{0} \leq \gamma_{z}<\alpha_{1}$ & yellow \\
\hline Small Curbs $(\sim 5 \mathrm{~cm})$ & $\alpha_{1} \leq \gamma_{z}<\alpha_{2}$ & orange \\
\hline Regular Curbs $(\sim 10 \mathrm{~cm})$ & $\alpha_{2} \leq \gamma_{z}<\alpha_{3}$ & red \\
\hline Big Obstacles & $\alpha_{3} \leq \gamma_{z} \leq 1$ & purple \\
\hline
\end{tabular}

range using morphological operations and contour analysis. The resulting masks are merged and re-filtered in order to get an image like the one shown in Figure 2(f). The use of fixed or empirical thresholds is then avoided given that the proposed function is adapted automatically for different scenes depending on the predominant curvature value.

\section{Increase Cloud Density}

The point cloud obtained from the stereo sensor is acceptably dense, while the LIDAR cloud is sparse given that the vertical resolution is limited to 0.4 degrees. As a consequence of that, the algorithm described in Section II-C cannot be applied directly. The cloud requires being dense enough. For that purpose, for every point the nearest neighbors (NN) are fitted to a surface. Then, new points are generated by drawing samples from the estimated surface. If the road surface is estimated using a polynomial, the resulting shape of small curbs is smoothed up and, consequently, those curbs are partially removed. In order to avoid this problem, an iterative algorithm is applied to align the sparse clouds from instant $t-n$ to $t$. As a result of that alignment, a dense cloud is created. This algorithm is denoted as Iterative Closest Point (ICP) [18], [19].

Data: point cloud $A=\left\{a_{1}, \ldots, a_{M}\right\}$, point cloud $B=\left\{b_{1}, \ldots, b_{N}\right\}$, initialized matrix T.

Result: The transformation matrix $\mathrm{T}$ which aligns $\mathrm{A}$ and $\mathrm{B}$.

while not converged do

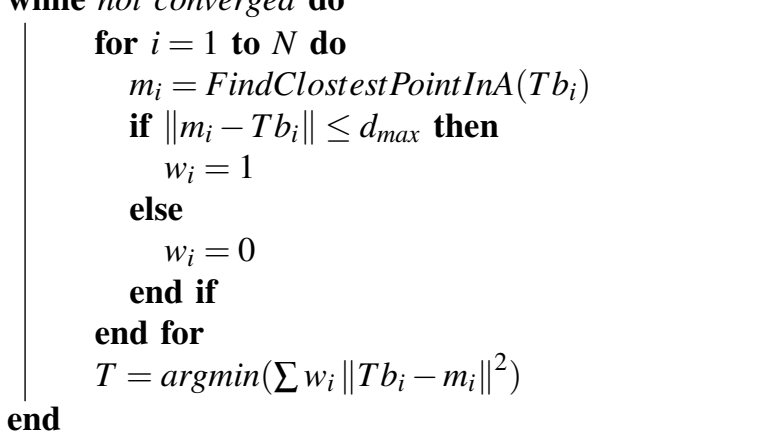

Algorithm 1: Standard ICP

The algorithm is illustrated in Algorithm 1. ICP minimizes the difference between two point clouds iteratively. The first one $A$ is kept fixed and the other one $B$ is warped to match the reference. If the clouds are close enough to each other the initial values for $T$ can be set to identity. For each iteration, every point in $B$ is transformed using the current transformation matrix $T$ and matched with the corresponding point in $A$. If the distance between the points is greater than $d_{\text {max }}$, the points are rejected. As depicted in Figure 3 , the ICP algorithm does not smooth the original data and small curbs 
can still be detected using with the algorithm explained in Section II-C.

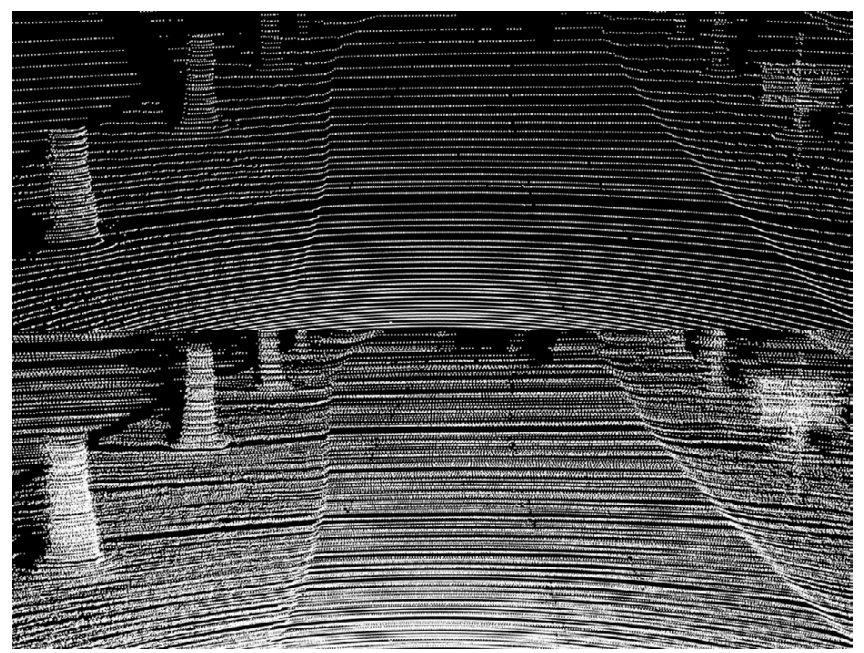

Fig. 3. Top: Single LIDAR scan. Bottom: Point cloud obtained with the Iterative Closest Point algorithm using the last 5 scans of the LIDAR.

\section{RESULTS}

The lateral accuracy was evaluated in road sequences from the KITTI dataset. In such dataset no ground-truth is provided for curbs detection. A contribution of this paper is the creation of a public dataset containing a number of sequences with manually labeled curbs. The ground-truth was manually annotated in the stereo image and also in the LIDAR data. The number of labeled sequences in our dataset is still low, although it will be gradually increased. The dataset is publicly available at www. isislab.es.

For the evaluation, straight lines are fitted to the detected curb points and also to the labeled ground-truth points. The estimated line and the ground truth are defined by the points $p$ and $q$ and the direction vectors $\vec{u}$ and $\vec{v}$ respectively. The accuracy is evaluated by computing equation 7, where $a$ and $b$ are the evaluated range, from 6 meters up to 20 meters. In urban scenarios the driving speed is usually low. Thus, we consider that an estimation range of 20 meters is enough for safe maneuvering in most cases.

$$
\begin{aligned}
l & =\frac{u_{y}}{u_{x}}\left(x-p_{x}\right)+p_{y} \\
\widehat{l} & =\frac{v_{y}}{v_{x}}\left(x-q_{x}\right)+q_{y} \\
R M S E & =\sqrt{\frac{1}{b-a} \int_{a}^{b}(l-\widehat{l})^{2} d x}
\end{aligned}
$$

In the case of the stereo data, the disparity images are computed using Semi Global Matching (SGM). By accumulating the latest 5 scans of the LIDAR, the density of the point cloud is enhanced, making it good enough for the curvature estimation algorithm. The density of the LIDAR cloud is increased using ICP, therefore some errors during

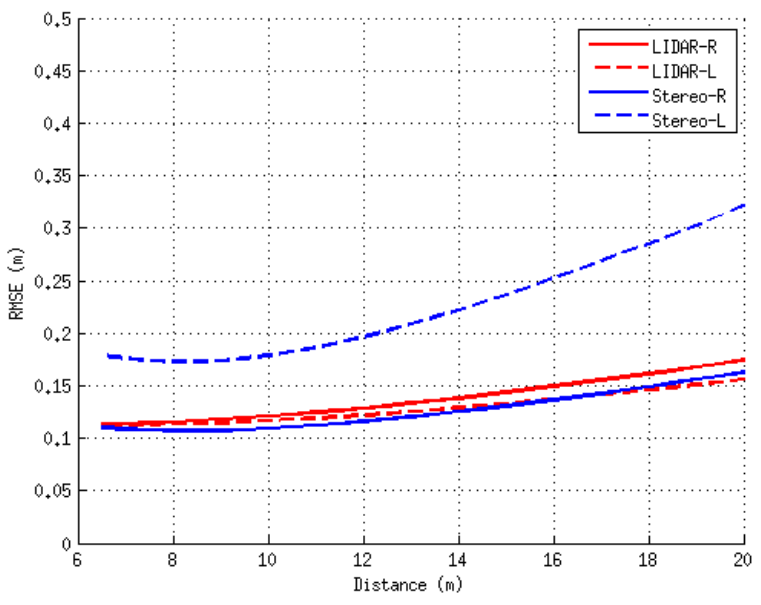

Fig. 4. Lateral RMSE obtained comparing ground truth and the result of the algorithm. The results for left and right curbs are separated to evaluate the performance individually.

the matching stage can yield point clouds with slightly worse quality than a dense stereo point cloud. In Figure 4, the lateral error is depicted. The LIDAR error is plotted in red and the error from the stereo is plotted in blue. The performance of our approach using the LIDAR data is quite similar both for the left and right curbs. In addition, the performance using the $3 \mathrm{D}$ data obtained from stereo vision is also similar to LIDAR for the right most curb. However, the lateral error on the left curb is significantly higher. By analyzing the disparity images in several sequences, we noted that the left part of the image provides noisy and unrealistic 3D information (see Figure 5) which can be caused by several factors (e.g., inaccurate calibration). As shown in Figure 4, when accurate disparity is available (right curb) the performance of the curvature estimation based on stereo vision is even better than the LIDAR because stereo-based point cloud is more dense than that obtained from the LIDAR. Therefore, we can state that the use of stereo cameras for ADAS in urban environments is a goodenough technology for accurate road curb detection. The curb detection algorithm presents a lateral RMSE of $12 \mathrm{~cm}$ in a range from 6 meters to 20 meters for the right most curb, while the value of RMSE on the left side is $22 \mathrm{~cm}$.

\section{CONCLUSIONS AND FUTURE WORKS}

In this paper a curb detection estimation algorithm based on $3 \mathrm{D}$ point clouds has been presented. The use of fixed or empirical thresholds is avoided given that the proposed function is adapted automatically for different road scenes depending on the predominant curvature value. A public dataset of manually labeled curbs has been made available at www.isislab.es. The algorithm presented in this paper has successfully been applied for the detection of straight and curved curbs.

However a geometric detection method is not enough to get a robust free space detection system due to the fact that some road limits have the same height as the road. 

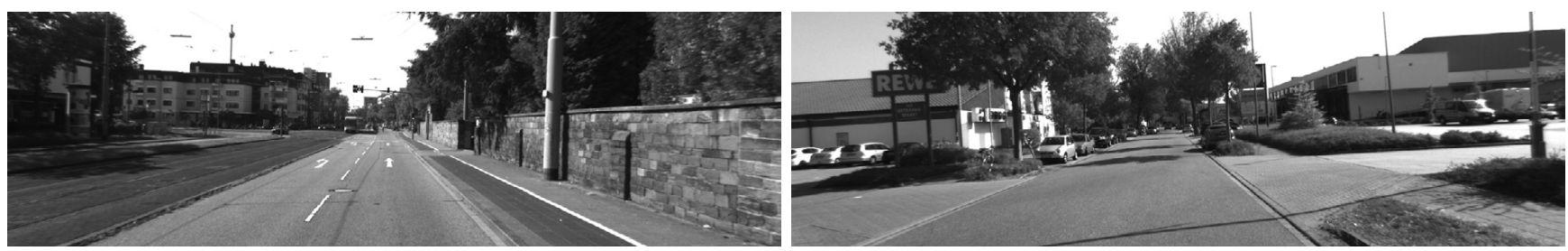

(a) Greyscale image
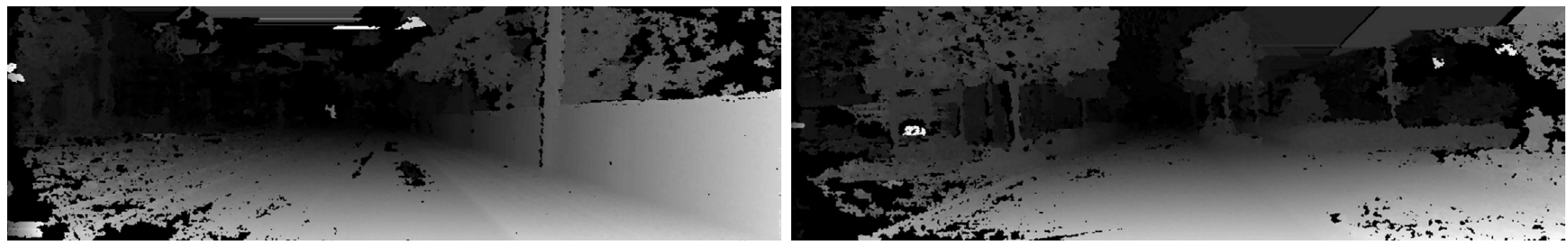

(b) Disparity image
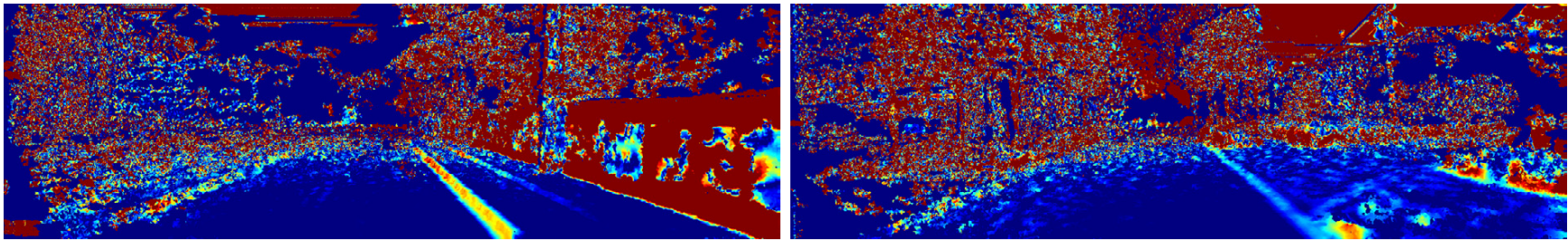

(c) Curvature estimation using stereo point cloud
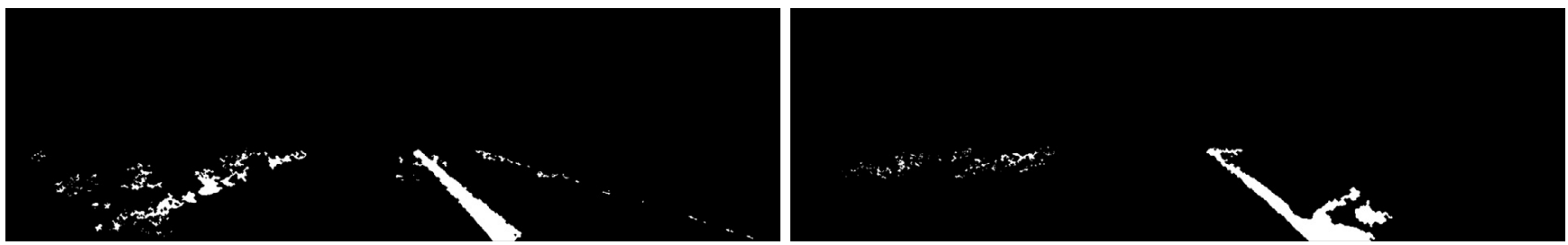

(d) Output of the curb detection algorithm for the stereo point cloud
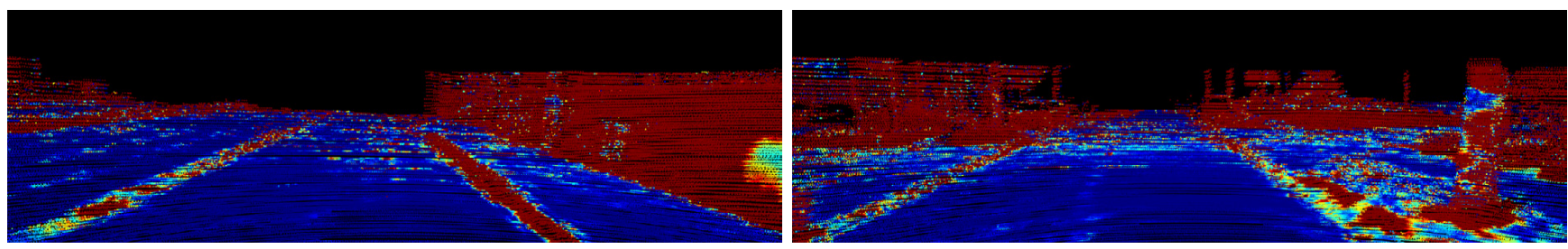

(e) Curvature estimation using LIDAR point cloud
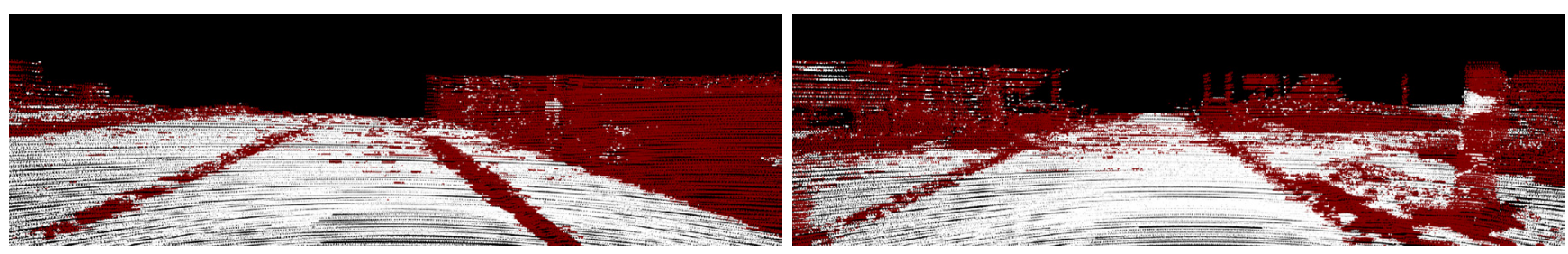

(f) Output of the curb detection algorithm for the LIDAR point cloud

Fig. 6. Comparison of curvature estimation. Results obtained on stereo vision are noisier than those obtained on LIDAR. 


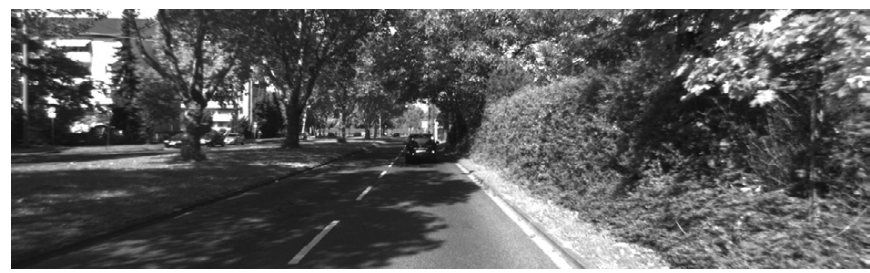

(a) Greyscale image of the analyzed frame

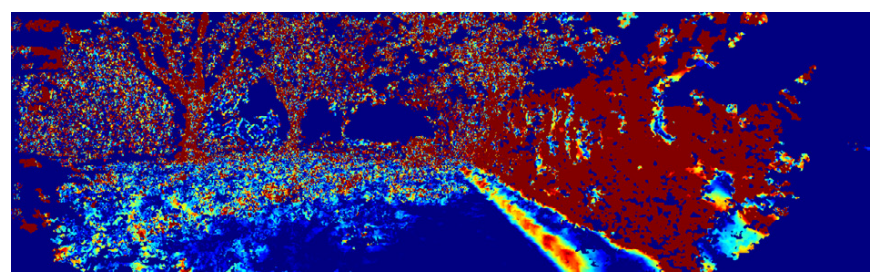

(b) Curvature estimation using 3D stereo data

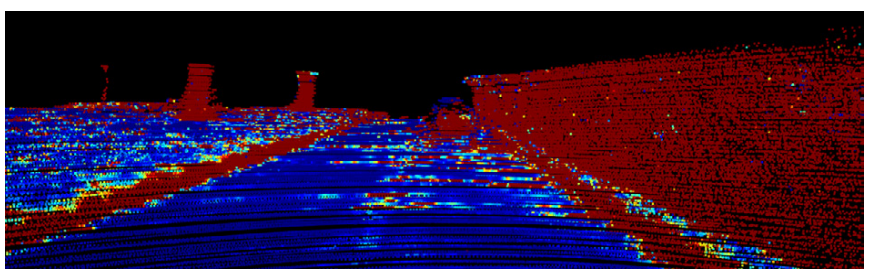

(c) Curvature estimation using LIDAR data

Fig. 5. Comparison of curvature estimation. Stereo calibration and mismatching errors produce wrong surface reconstruction on the left side of the image.

For this reason texture and color provide key information that complements and enhance the geometric reconstruction. As a future work, a new machine learning approach will include the geometric reconstruction presented in this paper together with texture and color information in order to detect the drivable area.

The proposed algorithm is compared using different sources for the $3 \mathrm{D}$ cloud data. Although the LIDAR cloud is more accurate than the stereo cloud, it is sparser. In order to improve the LIDAR cloud density, the Iterative Closest Point (ICP) has been applied. The density of the resulting cloud is similar to that of stereo vision systems, while preserving the accuracy of LIDAR sensors. Another advantage is the fact that laser-based reflectivity measurements are also available. The reflectivity is very useful to detect traffic signs and road markings during daytime and nighttime. In our future work, an algorithm for road marking detection on all-weather and all illumination conditions will be developed by jointly using LIDAR and cameras.

\section{ACKNOWLEDGMENTS}

This work was supported by the Spanish Ministry of Economy and Competitiveness under Research Grant ONDA-FP TRA2011-27712-C02-02, and by the General Traffic Division of Spain under Research Grant DISADAPT SPIP20141300

\section{REFERENCES}

[1] M. A. Sotelo, F. J. Rodriguez, and L. Magdalena, "Virtuous: visionbased road transportation for unmanned operation on urban-like sce- narios," IEEE Transactions on Intelligent Transportation Systems, vol. 5, no. 2, pp. 69-83, June 2004.

[2] M. A. Sotelo, F. J. Rodriguez, L. Magdalena, L. M. Bergasa, and L. Boquete, "A color vision-based lane tracking system for autonomous driving on unmarked roads," Autonomous Robots, vol. 16, no. 1, pp. 95-116, 2004.

[3] G. Finlayson, S. Hordley, C. Lu, and M. Drew, "On the removal of shadows from images," IEEE Transactions on Pattern Analysis and Machine Intelligence, vol. 28, no. 1, pp. 59-68, Jan 2006.

[4] J. Alvarez and A. Lopez, "Road detection based on illuminant invariance," IEEE Transactions on Intelligent Transportation Systems, vol. 12, no. 1, pp. 184-193, March 2011.

[5] J. Fritsch, T. Kuhnl, and F. Kummert, "Monocular road terrain detection by combining visual and spatial information," IEEE Transactions on Intelligent Transportation Systems, vol. PP, no. 99, pp. 1-11, 2014.

[6] C. Fernandez, M. Gavilan, D. Llorca, I. Parra, R. Quintero, A. Lorente, L. Vlacic, and M. Sotelo, "Free space and speed humps detection using lidar and vision for urban autonomous navigation," in Intelligent Vehicles Symposium (IV), 2012 IEEE, June 2012, pp. 698-703.

[7] C. Guo, T. Yamabe, and S. Mita, "Robust road boundary estimation for intelligent vehicles in challenging scenarios based on a semantic graph," in IEEE Intelligent Vehicles Symposium (IV), June 2012, pp. 37-44.

[8] C. Guo, S. Mita, and D. McAllester, "Robust road detection and tracking in challenging scenarios based on markov random fields with unsupervised learning," IEEE Transactions on Intelligent Transportation Systems, vol. 13, no. 3, pp. 1338-1354, Sept 2012.

[9] B. Ranft and T. Strauss, "Modeling arbitrarily oriented slanted planes for efficient stereo vision based on block matching," in Intelligent Transportation Systems (ITSC), 2014 IEEE 17th International Conference on, Oct 2014, pp. 1941-1947.

[10] J. Siegemund, D. Pfeiffer, U. Franke, and W. Frstner, "Curb reconstruction using conditional random fields," in IEEE Intelligent Vehicles Symposium (IV), June 2010, pp. 203-210.

[11] J. Siegemund, U. Franke, and W. Frstner, "A temporal filter approach for detection and reconstruction of curbs and road surfaces based on conditional random fields," in IEEE Intelligent Vehicles Symposium (IV), June 2011, pp. 637-642.

[12] J. Ziegler, P. Bender, M. Schreiber, H. Lategahn, T. Strauss, C. Stiller, T. Dang, U. Franke, N. Appenrodt, C. Keller, E. Kaus, R. Herrtwich, C. Rabe, D. Pfeiffer, F. Lindner, F. Stein, F. Erbs, M. Enzweiler, C. Knoppel, J. Hipp, M. Haueis, M. Trepte, C. Brenk, A. Tamke, M. Ghanaat, M. Braun, A. Joos, H. Fritz, H. Mock, M. Hein, and E. Zeeb, "Making bertha drive?an autonomous journey on a historic route," IEEE Intelligent Transportation Systems Magazine, vol. 6, no. 2, pp. 8-20, Summer 2014.

[13] H. Hirschmuller, "Accurate and efficient stereo processing by semiglobal matching and mutual information," in Proceedings of the 2005 IEEE Computer Society Conference on Computer Vision and Pattern Recognition (CVPR'05). Washington, DC, USA: IEEE Computer Society, 2005, pp. 807-814.

[14] A. Geiger, P. Lenz, C. Stiller, and R. Urtasun, "Vision meets robotics: The kitti dataset," International Journal of Robotics Research (IJRR), 2013.

[15] M. Pauly, M. Gross, and L. Kobbelt, "Efficient simplification of pointsampled surfaces," in Visualization, 2002. VIS 2002. IEEE, Nov 2002, pp. $163-170$.

[16] A. Hata, F. Osorio, and D. Wolf, "Robust curb detection and vehicle localization in urban environments," in Intelligent Vehicles Symposium Proceedings, 2014 IEEE, June 2014, pp. 1257-1262.

[17] C. Fernandez, R. Izquierdo, D. Llorca, and M. Sotelo, "Road curb and lanes detection for autonomous driving on urban scenarios," in Intelligent Transportation Systems (ITSC), 2014 IEEE 17th International Conference on, Oct 2014, pp. 1964-1969.

[18] S. Rusinkiewicz and M. Levoy, "Efficient variants of the icp algorithm," in 3-D Digital Imaging and Modeling, 2001. Proceedings. Third International Conference on, 2001, pp. 145-152.

[19] M. Greenspan and M. Yurick, "Approximate k-d tree search for efficient icp," in 3-D Digital Imaging and Modeling, 2003. 3DIM 2003. Proceedings. Fourth International Conference on, Oct 2003, pp. 442448. 